\title{
Feasibility and acceptability of family administration of delirium detection tools in the intensive care unit: a patient-oriented pilot study
}

\author{
Karla D. Krewulak PhD, Bonnie G. Sept, Henry T. Stelfox MD PhD, E.W. Ely MD MPH, \\ Judy E. Davidson DNP RN, Zahinoor Ismail MD, Kirsten M. Fiest PhD
}

Abstract

Background: Family-administered delirium detection tools may serve as valuable diagnostic adjuncts because family caregivers may be better able than providers to detect changes in patient cognition and behaviour from pre-illness levels of functioning. The aim of this pilot study was to assess the feasibility and acceptability of family-administered tools to detect delirium in critically ill patients.

Methods: In this single-centre pilot tool validation study conducted in August and September 2017, eligible family caregivers used the Family Confusion Assessment Method (FAM-CAM) and the Sour Seven questionnaire to detect delirium during the patient's intensive care unit (ICU) stay. We calculated descriptive statistics for all study variables. Patients and family caregivers were involved as research partners throughout the study. A patient-orient research approach was taken, engaging patients and family caregivers as full partners.

Results: Of 141 patients admitted to the ICU, 75 were eligible, of whom 53 were approached; 21 patients (40\%), 23/38 family caregivers $(60 \%)$ and $17 / 38$ dyads (i.e., patient and family caregiver enrolled together) $(45 \%)$ consented to participate. The most common reason for nonenrolment was refusal by the family, who commonly reported feeling overwhelmed. The completion rate for the FAMCAM and Sour Seven questionnaire was 74\% (17/23). Among 13 dyads, family caregivers detected delirium in 5 patients (38\%) using the FAM-CAM, and delirium or possible delirium in 8 patients $(62 \%)$ using the Sour Seven questionnaire, whereas trained research assistants detected delirium in 8 patients (62\%) using the Confusion Assessment Method for the Intensive Care Unit 7 and the Richmond Agitation-Sedation Scale ( $\kappa$ coefficient for agreement between the former and the FAM-CAM and Sour Seven questionnaire 0.62 and 0.85 , respectively).

Interpretation: Administration of the FAM-CAM and Sour Seven questionnaire by family caregivers to detect delirium in the ICU is feasible and acceptable, although, as with most family engagement strategies, it was not desired by all. Results from this pilot study support a definitive study with a larger sample to enable calculation of inferential statistics, but additional recruitment strategies are necessary to improve the response rate. Trial registration: Clinicaltrials.gov, no. NCT03379129.

Plain language summary: Patients with delirium have changes in their thinking and are often confused and cannot pay attention. About half of patients in an intensive care unit (ICU) have delirium during their stay. Research has shown that patients with delirium are more likely to die or to have long-term brain problems, including posttraumatic stress disorder, depression and other mental health issues, than those without delirium. Although nurses and doctors have tools to measure delirium in the ICU, it can be hard to identify and, in some cases, may be missed. Family members may be the first to notice that their loved ones have changes in their thinking or cannot pay attention. There are tools called the Family Confusion Assessment Method (FAM-CAM) and Sour Seven questionnaire that can be used by family members to detect delirium. However, neither of these tools has been used in an ICU. By doing this study, we were able to show that these tools can be used by family members to measure delirium in the ICU. The results from this study may lead to a change in policy that would involve partnering with family members to improve the diagnosis of delirium in the ICU. In turn, this would improve patient and family care and outcomes in the ICU.

Competing interests: None declared. The developer of the Confusion Assessment Method for the Intensive Care Unit, E.W. Ely, did not perform the evidence analysis of the study.

This article has been peer reviewed.

Correspondence to: Kirsten Fiest, kmfiest@ucalgary.ca

CMAJ Open 2019. DOI:10.9778/cmajo.20180123 


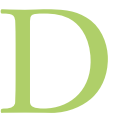

elirium is an acute-onset brain disorder marked by inattention and confusion. ${ }^{1}$ It affects $32 \%$ of patients admitted to intensive care units (ICUs) ${ }^{2}$ and up to $80 \%$ of patients receiving mechanical ventilation. ${ }^{3,4}$ Patients in the ICU may experience several influences (e.g., pain, sedatives, dehydration) that confer an increased risk of delirium. ${ }^{5}$ Delirium is associated with negative outcomes in survivors of critical illness, ${ }^{6,7}$ and family caregivers of critically ill patients may experience stress-related complications after patient discharge. ${ }^{8-10}$ Family caregivers at the bedside may notice features of delirium that a member of the ICU care team may miss. Delirium detection by the family may help both patients and families through earlier and more accurate recognition of delirium and meaningful family involvement, with the potential for better patient and family outcomes. A recent systematic review ${ }^{11}$ identified 2 family-caregiver-administered delirium detection tools amenable for use in the ICU: the Family Confusion Assessment Method (FAM-CAM) ${ }^{12}$ and the Sour Seven questionnaire. ${ }^{13}$

The Facilitated Sensemaking model of family member engagement in critical care posits that, by providing opportunities for family caregivers to participate in communication, decision-making and bedside care, a sense of purpose derived from active participation in care can serve as a protective mechanism to reduce stress-related complications among family caregivers of critically ill patients. ${ }^{14,15}$ As such, patients admitted to the ICU and family caregivers were engaged as research partners to inform all elements of the current study. The primary objective of the study was to assess the feasibility and acceptability of using 2 family-caregiver-administered delirium detection tools (FAM-CAM and Sour Seven questionnaire) among critically ill patients. This pilot study will identify modifications needed in the design of a larger, hypothesis-testing study, the larger Family ICU Delirium Detection Study (ClinicalTrials.gov, no. NCT03379129).

\section{Methods}

\section{Setting}

Between Aug. 9 and Sept. 21, 2017, we recruited a sample of consecutive patients and family caregivers from the 28-bed general systems adult ICU at Foothills Medical Centre, Calgary (catchment population 1.5 million).

\section{Sample and recruitment}

Inclusion criteria for patients were: 1) age 18 years or more, 2) Richmond Agitation-Sedation Scale (RASS) score -3 or higher, $\left.{ }^{16} 3\right)$ no primary direct brain injury, 4 ) able to communicate with the study team (i.e., able to understand English and no hearing or visual impairment that precluded communication), 5) able to provide informed consent or have a surrogate decision-maker present and 6) expected to remain in the ICU long enough to allow for assessments to be completed at least once. Family caregivers were defined as anyone who provided physical or emotional support to a patient (e.g., a relative, friend or formal caregiver) and who had knowledge of the patient before ICU admission. Inclusion criteria for family caregivers were: 1) age 18 years or more, 2) able to understand both written and spoken English and 3) able to provide informed consent.

\section{Data collection}

All consecutive, eligible patients and their family caregivers were asked by the bedside registered nurse whether a research associate (K.D.K.) or patient researcher (B.G.S.) could approach them to discuss a research opportunity (Appendix 1, available at www.cmajopen.ca/content/7/2/E294/suppl/DC1). If both an eligible patient (through direct or surrogate consent) and family caregiver consented, they were enrolled as a dyad. Participants also included separate patients or family caregivers, so as to not exclude anyone. If the family caregiver was not present at the time of patient recruitment, the research associate followed up until the patient was discharged or transferred from the ICU. Patients who provided consent or their surrogate completed a demographic questionnaire (Appendix 2, available at www.cmajopen.ca/content/7/2/ E294/suppl/DC1). Family caregivers completed 2 paper questionnaires designed to detect delirium within 24 hours of meeting eligibility criteria for the study: the 11-item FAM-CAM ${ }^{12}$ and the 7-item Sour Seven. ${ }^{13}$ A description of these tools is provided in Appendix 3 (available at www. cmajopen.ca/content/7/2/E294/suppl/DC1). A blinded member of the study team (K.D.K. or B.G.S.) stayed with the family until the questionnaires were completed or explained the questionnaires and left them for the family to complete afterward, according to family preference. The questionnaires took less than 10 minutes to complete.

To compare family-administered delirium screening with a delirium screening tool previously validated in the ICU, ${ }^{3,17,18}$ a trained research associate (K.D.K.) or 1 of 2 research assistants) completed independent blinded Confusion Assessment Method for the Intensive Care Unit 7 (CAM-ICU-7) and RASS assessments (piloted for the full study) at the same time twice daily (between 0900 and 1100, and 1400 and 1600) for a maximum of 5 business days (i.e., maximum of 10 assessments). The training is described in Appendix 4 (available at www.cmajopen.ca/content/7/2/E294/suppl/DC1). The CAM-ICU-7 is a delirium screening tool that allows for dichotomous (delirium/no delirium) and continuous (no, mild to moderate, or severe delirium) measurement of delirium. It has high internal consistency (Cronbach $\alpha=0.85$ ) and good construct validity (correlation coefficient 0.64 ) (Appendix 3). ${ }^{17}$ The RASS measures the level of patient sedation or agitation and is used together with the CAM-ICU-7 to identify delirium subtype. ${ }^{19}$ The RASS has excellent interrater reliability $(\kappa=0.91,95 \%$ confidence interval $[\mathrm{CT}] 0.86-0.95)$ and high construct validity (correlation coefficient 0.91 ). ${ }^{16,20}$ Both tools require less than 1 minute to complete.

A trained ICU research nurse with experience assessing delirium in critically ill patients performed reference standard assessments of delirium (piloted for the full study) once daily between 1400 and 1600 for a maximum of 5 days (see Appendix 4 for a description of the training). The research nurse 
used a standardized assessment form (developed by K.D.K., Z.I. and an ICU research nurse) operationalizing Diagnostic and Statistical Manual of Mental Disorders, Fifth Edition criteria ${ }^{1}$ to identify delirium (Appendix 5, available at www.cmajopen. ca/content/7/2/E294/suppl/DC1). The research nurse gathered information from the patient, family caregiver(s), bedside nurse, physician and electronic medical record to complete the standardized assessment form (while remaining blind to previous assessments of delirium). The reference assessment took 5 minutes to complete. The research assistants and ICU research nurse took field notes, which were discussed weekly during team meetings. Regular daily reference standard assessments did not occur because this standardized form was piloted during the study.

\section{Patient engagement}

Three patients were engaged as full partners. Patient partner engagement was guided by the Canadian Institutes of Health Research Strategies for Patient-Oriented Research Patient Engagement Framework. ${ }^{21,22}$ During a 2-day stakeholder engagement meeting (Nov. 17-18, 2016), the study team sought to inform and consult ICU patients $(n=3)$, family caregivers $(n=4)$, researchers $(n=19)$ and care providers $(n=10)$ on family-centred research priorities in the ICU. Patients and family caregivers were invited to the meeting through existing relationships with the study team ${ }^{23}$ and referral from engaged patients and family caregivers. There were no criteria for participation other than lived ICU experience as a patient or family caregiver. Through a series of prioritization activities, the meeting participants identified delirium prevention, detection and management as an area where family caregivers could be meaningfully engaged in the care of critically ill patients. Patients and family caregivers participated as equal partners in the meeting, including delivering presentations and facilitating discussion. Two patient partners from the meeting codesigned the research questions and proposals for the current study during inperson meetings. They developed the research questions using the PICO (Population, Intervention, Comparison, Outcome) framework. The procedure for approaching patients and family caregivers was discussed, and notes were taken. The notes were transcribed into a study protocol and study materials, which were reviewed by the patient partners. The patient partners were coapplicants on successful funding applications. Before pilot study recruitment, the patient partners communicated the study to the ICU team through multiple hallway "huddles." Neither patient partner wished to be involved with participant recruitment or data entry, but they were updated on the pilot study progress regularly via email. Near the end of the pilot study, a third patient partner (B.G.S.) joined our team and assisted with recruitment and questionnaire completion. She approached patients and family caregivers about the study, obtained consent and, in some cases, stayed while they completed the study questionnaires. A research associate (K.D.K.) trained B.G.S. (Appendix 4) and was available to answer questions, receive feedback and provide support.

\section{Data analysis}

We assessed feasibility of recruitment by the number of eligible participants who were approached, recruited and enrolled. Based on our experience at this ICU and the recruitment rates in delirium studies in adult ICUs, ${ }^{24,25}$ we determined a feasible recruitment rate of $33 \%$. We assessed the feasibility of delirium assessments by comparing the number of eligible assessments to the number of delirium assessments for which we had complete data. Reasons for missing assessments were recorded.

We present feasibility (i.e., a recruitment rate, response rate), acceptability (delirium assessment procedures) and participant characteristics using descriptive statistics calculated in Stata (StataCorp). We categorized delirium using each of the CAM-ICU-7 (no delirium [0-2], mild to moderate delirium [3-5], severe delirium [6-7]), FAM-CAM (all features of delirium present/absent) and Sour Seven (probable delirium [>9], possible delirium [> 4) tools. We calculated the Cohen $\kappa$ as a measure of agreement, where $0.40-0.75$ = fair to good and greater than $0.75=$ excellent. $^{26}$

\section{Ethics approval}

The study was approved by the Conjoint Health Research Ethics Board at the University of Calgary.

\section{Results}

Participant recruitment and reasons for exclusion are depicted in Figure 1. A total of 53 (71\%) of 75 eligible patients were approached. The overall recruitment rates for approached patients and family caregivers were 40\% (21/53) and 60\% (23/38), respectively. Of the 53 eligible patients, 38 (72\%) had a family caregiver present at the time of recruitment, of whom 17 (45\%) were enrolled as part of a dyad. Among the 16 eligible patients whose families were approached for surrogate consent but did not participate, 12 caregivers (75\%) reported being overwhelmed or requested time to think about participation. No participants withdrew from the study or indicated that the study was a burden once enrolled. Participant characteristics are listed in Table 1.

Of the 23 family caregivers, 17 (74\%) completed the FAM-CAM and Sour Seven questionnaire (Table 2). Some caregivers overwhelmed by the consent process asked to complete the study questionnaires at a later time. However, the variability and unpredictability of caregivers' visiting times made it difficult to follow up. When the questionnaires were completed in the presence of a research associate/patient partner immediately after the consent process, the completion rate was $100 \%(12 / 12)$. No family caregiver indicated that the questionnaires were too difficult to complete.

Seventy-seven percent (105/137) of CAM-ICU-7 and RASS assessments were completed. The most common reason for missed assessments was inability to assess the patient during the assessment time frame (15/32 [47\%]). Two of the assessments that were declined by the family caregiver involved the same person, who was overwhelmed.

The CAM-ICU-7, FAM-CAM and Sour Seven questionnaire were completed for 13 dyads. Delirium was detected in 
$8(62 \%)$ of the 13 patients, as indicated by at least 1 positive CAM-ICU-7 assessment. Family caregivers detected delirium in 5 patients $(38 \%)$ using the FAM-CAM, and delirium or possible delirium in 8 patients $(62 \%)$ using the Sour Seven questionnaire. The $\kappa$ coefficient for agreement between the CAM-ICU-7 and the FAM-CAM and Sour Seven questionnaire was 0.62 and 0.85 , respectively.

\section{Interpretation}

This pilot tool validation study showed that family administration of delirium detection tools is feasible and acceptable in the ICU. A $100 \%$ response rate was achieved when the FAM-CAM and Sour Seven questionnaire were completed immediately after enrolment. These results support a larger study to validate these family-administered delirium detection tools in critically ill patients. We piloted the reference stan- dard assessment and confirmed that it is acceptable for validating the 2 family-administered delirium detection tools in future studies.

The importance of family involvement in the care of critically ill patients has been explored in several studies. ${ }^{10,14,15,27,28}$ For example, there was a decrease in symptoms of anxiety and depression in families who witnessed cardiopulmonary resuscitation ${ }^{14,15}$ and those who received information on what to expect at the end of life. ${ }^{28}$ Delirium detection by the family has the potential to produce similar positive effects. To assess the acute onset and fluctuating course of delirium, the Family ICU Delirium Detection Study protocol will include daily familyadministered delirium assessments for up to 5 consecutive days instead of once overall. In addition, we will use a questionnaire eliciting family caregiver demographic characteristics to explore the effects of family caregiver age, sex, biological sex and education on delirium detection by the family.

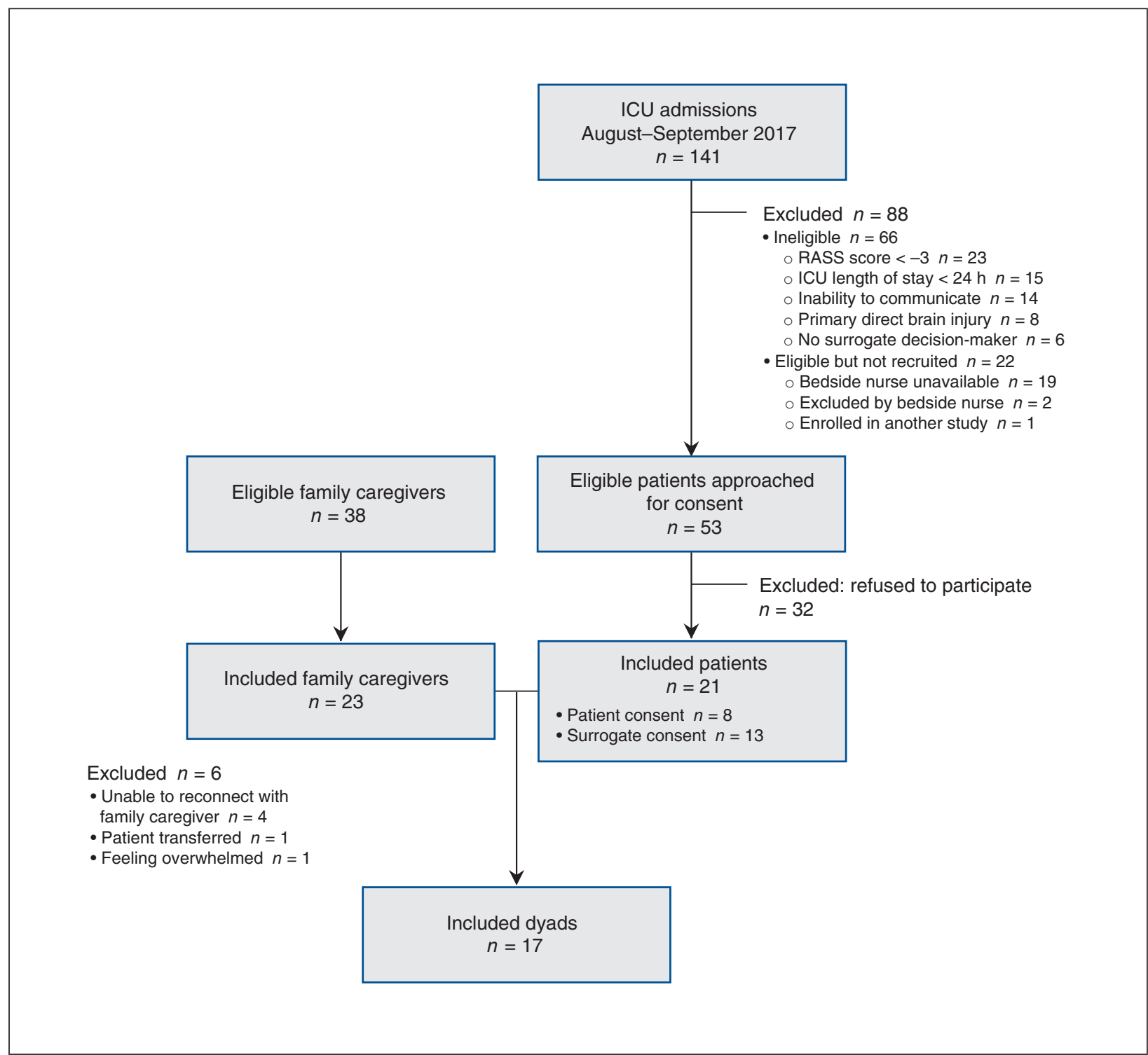

Figure 1: Flow diagram showing participant selection. Note: ICU = intensive care unit, RASS = Richmond Agitation-Sedation Scale. 


\begin{tabular}{|c|c|c|}
\hline \multicolumn{3}{|c|}{$\begin{array}{l}\text { Table 1: Demographic characteristics of included patients } \\
\text { and family caregivers }\end{array}$} \\
\hline \multirow[b]{2}{*}{ Characteristic } & \multicolumn{2}{|c|}{ No. $(\%)$ of participants* } \\
\hline & $\begin{array}{l}\text { Patients } \\
n=21\end{array}$ & $\begin{array}{c}\text { Family } \\
\text { caregivers } \\
n=23\end{array}$ \\
\hline Age, yr, mean \pm SD (range) & $48.0 \pm 17.6(20-84)$ & - \\
\hline Female sex & $8(38)$ & $14(61)$ \\
\hline \multicolumn{3}{|l|}{ Education } \\
\hline Some high school & $3(14)$ & - \\
\hline High school graduate & $2(10)$ & - \\
\hline $\begin{array}{l}\text { Some university/college } \\
\text { (no degree) }\end{array}$ & $3(14)$ & - \\
\hline Bachelor's degree & $2(10)$ & - \\
\hline Other & $3(14)$ & - \\
\hline Missing & $8(38)$ & - \\
\hline \multicolumn{3}{|l|}{ Relationship to patient } \\
\hline Spouse/common-law & - & $11(48)$ \\
\hline Daughter & - & $4(17)$ \\
\hline Son & - & $2(9)$ \\
\hline Mother & - & $2(9)$ \\
\hline Father & - & $2(9)$ \\
\hline Sibling & - & $2(9)$ \\
\hline $\begin{array}{l}\text { Note: } \mathrm{SD}=\text { standard deviation. } \\
{ }^{\star} \text { Except where noted otherwise. }\end{array}$ & & \\
\hline
\end{tabular}

Table 2: Completion of family-administered delirium questionnaires and patient assessments

\begin{tabular}{|c|c|c|}
\hline \multirow[b]{2}{*}{ Questionnaire/assessment } & \multicolumn{2}{|c|}{ No. (\%) of assessments } \\
\hline & Completed & $\begin{array}{c}\text { Not } \\
\text { completed }\end{array}$ \\
\hline \multicolumn{3}{|l|}{ Family-administered $(n=23)$} \\
\hline FAM-CAM, Sour Seven & $17(74)$ & $6(26)$ \\
\hline \multicolumn{3}{|l|}{ Reason not completed } \\
\hline $\begin{array}{l}\text { Unable to reconnect with family } \\
\text { caregiver }\end{array}$ & & $4(67)$ \\
\hline Patient transferred & & $1(17)$ \\
\hline Family caregiver overwhelmed & & $1(17)$ \\
\hline \multicolumn{3}{|l|}{ Patient assessments $(n=137)$} \\
\hline CAM-ICU-7, RASS & $105(77)$ & $32(23)$ \\
\hline \multicolumn{3}{|l|}{ Reason not completed } \\
\hline Unable to assess patient & & $15(47)$ \\
\hline Patient not eligible (RASS score $<-3$ ) & & $10(31)$ \\
\hline Nurse discretion & & $4(12)$ \\
\hline Family declined assessment & & $3(9)$ \\
\hline \multicolumn{3}{|c|}{$\begin{array}{l}\text { Note: CAM-ICU-7 = Confusion Assessment Method for the Intensive Care } \\
\text { Unit 7, FAM-CAM = Family Confusion Assessment Method, RASS = Richmond } \\
\text { Agitation-Sedation Scale. }\end{array}$} \\
\hline
\end{tabular}

\section{Limitations}

With only 17 completed questionnaires, this descriptive study was not powered to test the validity or reliability of the tools. Neither tool has been validated in the ICU context. The completion rate was lower than expected for the FAM-CAM and Sour Seven questionnaire. To ensure an improved completion rate in the full study, we hired a patient partner (B.G.S.) to approach bedside nurses, enrol participants and assist family caregivers with the completion of the questionnaires. We expect this will increase the completion rate because this family caregiver has the lived experience, perseverance and communication skills that are necessary to make the consent process less overwhelming. Another limitation is misinterpretation of delirium by family caregivers when the patient's symptoms are unrelated to delirium (e.g., depression, effects of sedative medications). Level of consciousness may be a confounding factor when assessing delirium, and symptoms of psychomotor slowing should not be considered present if due to sedation..$^{29}$ In the full study, we will record the dosage and type of sedative administered as well as the level of sedation based on the RASS and will stratify the results by sedation level. We will use aggregate data from all patients during the enrolment period to determine how the included participants compare with the entire eligible sample. It is possible that the ICU research nurse performing the reference standard assessments was not blinded to previous delirium status or RASS. The full study will include a blinding assessment with each reference standard assessment, and we will conduct a sensitivity analysis to determine whether lack of blinding influences study outcomes. In addition, $10 \%$ of reference standard assessments will be completed by 2 research nurses, blinded to one another's assessments for the purpose of assessing interrater reliability. Finally, enrolment at a single centre may limit the generalizability of our results. However, the Foothills Medical Centre has the largest ICU in southern Alberta and serves a diverse group of patients.

\section{Lessons learned from patient engagement}

There were many aspects of this pilot study that worked well. The goal of the Family ICU Delirium Detection Study is to partner with patients and families at the bedside. As such, patient partners codesigned the study protocol, recruitment scripts and consent forms. Their lived experience was invaluable in the design of this study to ensure that the study protocol, the manner in which we approached potential participants and consent forms were acceptable for patients in the ICU and their family caregivers. There are several challenges that will be addressed before the full study. Our research team has experience with engaging and training patient partners in ICU research, ${ }^{23}$ but there were lessons learned from working with patient partners in this study. Patient partners expressed frustration with the constraints of the research process, including the ethical and privacy considerations of clinical research. The successful inclusion of patient partners should include appropriate study-specific training and adequate supervision to ensure that they remain within the ethical boundaries of research. This includes access to the research team for information and scheduled refresher sessions to review study procedures. At the end 
of the full study, we will evaluate our patient engagement process using the Public and Patient Engagement Evaluation $\mathrm{Tool}^{30}$ to learn from the patient partners' experiences.

\section{Conclusion}

This pilot tool validation study showed the feasibility and acceptability of family detection of delirium in patients in the ICU. A full study will determine the reliability and validity of the family-administered delirium detection tools. The full study will include a qualitative component exploring the perspectives of family caregivers and the ICU team on delirium detection by the family. The overall aim is to improve delirium detection, which, in turn, may improve both patient and family outcomes.

\section{References}

1. Diagnostic and statistical manual of mental disorders. 5th edition. Arlington (VA): American Psychiatric Association; 2013.

2. Salluh JI, Wang H, Schneider EB, et al. Outcome of delirium in critically ill patients: systematic review and meta-analysis. BM7 2015;350:h2538.

3. Ely EW, Inouye SK, Bernard GR, et al. Delirium in mechanically ventilated patients: validity and reliability of the confusion assessment method for the intensive care unit (CAM-ICU). 7AMA 2001;286:2703-10.

4. Ely EW, Shintani A, Truman B, et al. Delirium as a predictor of mortality in mechanically ventilated patients in the intensive care unit. $7 A M A 2004 ; 291$ : 1753-62.

5. Zaal IJ, Devlin JW, Peelen LM, et al. A systematic review of risk factors for delirium in the ICU. Crit Care Med 2015;43:40-7.

6. Needham DM, Davidson J, Cohen H, et al. Improving long-term outcomes after discharge from intensive care unit: report from a stakeholders' conference. Crit Care Med 2012;40:502-9.

7. Rawal G, Yadav S, Kumar R. Post-intensive care syndrome: an overview. 7 Transl Int Med 2017;5:90-2

8. Davidson JE, Jones C, Bienvenu OJ. Family response to critical illness: postintensive care syndrome-family. Crit Care Med 2012;40:618-24.

9. Schmidt M, Azoulay E. Having a loved one in the ICU: the forgotten family. Curr Opin Crit Care 2012;18:540-7.

10. Cameron JI, Chu LM, Matte A, et al. One-year outcomes in caregivers of critically ill patients. N Engl 7 Med 2016;374:1831-41.

11. Rosgen B, Krewulak K, Demiantschuk D, et al. Validation of caregivercentered delirium detection tools: a systematic review. 7 Am Geriatr Soc 2018; 66:1218-25.

12. Steis MR, Evans L, Hirschman KB, et al. Screening for delirium using family caregivers: convergent validity of the Family Confusion Assessment Method and interviewer-rated Confusion Assessment Method. 7 Am Geriatr Soc 2012;60:2121-6.

13. Shulman RW, Kalra S, Jiang JZ. Validation of the Sour Seven Questionnaire for screening delirium in hospitalized seniors by informal caregivers and untrained nurses. BMC Geriatr 2016;16:44.

14. Jabre P, Belpomme V, Azoulay E, et al. Family presence during cardiopulmonary resuscitation. $N$ Engl 7 Med 2013;368:1008-18.

15. Jabre P, Tazarourte K, Azoulay E, et al. Offering the opportunity for family to be present during cardiopulmonary resuscitation: 1-year assessment. Intensive Care Med 2014;40:981-7.

16. Sessler CN, Gosnell MS, Grap MJ, et al. The Richmond Agitation-Sedation Scale: validity and reliability in adult intensive care unit patients. Am 7 Respir Crit Care Med 2002;166:1338-44.

17. Khan BA, Perkins AJ, Gao S, et al. The Confusion Assessment Method for the ICU-7 Delirium Severity Scale: a novel delirium severity instrument for use in the ICU. Crit Care Med 2017;45:851-7.

18. Ely EW, Margolin R, Francis J, et al. Evaluation of delirium in critically ill patients: validation of the Confusion Assessment Method for the Intensive Care Unit (CAM-ICU). Crit Care Med 2001;29:1370-9.

19. Peterson JF, Pun BT, Dittus RS, et al. Delirium and its motoric subtypes: a study of 614 critically ill patients. 7 Am Geriatr Soc 2006;54:479-84.
20. Ely EW, Truman B, Shintani A, et al. Monitoring sedation status over time in ICU patients: reliability and validity of the Richmond Agitation-Sedation Scale (RASS). 7AMA 2003;289:2983-91.

21. Strategy for patient-oriented research - patient engagement framework. Ottawa: Canadian Institutes of Health Research; modified 2014. Available: www.cihr-irsc.gc.ca/e/48413.html (accessed 2019 Feb. 11).

22. Staniszewska S, Brett J, Simera I, et al. GRIPP2 reporting checklists: tools to improve reporting of patient and public involvement in research. BM7 2017; 358: j3453.

23. Gill M, Bagshaw SM, McKenzie E, et al. Patient and family member-led research in the intensive care unit: a novel approach to patient-centered research. PLoS One 2016;11:e0160947.

24. Chlan LL, Weinert CR, Heiderscheit A, et al. Effects of patient-directed music intervention on anxiety and sedative exposure in critically ill patients receiving mechanical ventilatory support: a randomized clinical trial. $7 A M A 2013 ; 309$ : 2335-44.

25. Khan SH, Wang S, Harrawood A, et al. Decreasing Delirium through Music (DDM) in critically ill, mechanically ventilated patients in the intensive care unit: study protocol for a pilot randomized controlled trial. Trials 2017;18:574.

26. Fleiss JL. Statistical methods for rates and proportions. 2nd edition. New York: John Wiley \& Sons; 1981.

27. Schneiderman LJ, Gilmer T, Teetzel HD, et al. Effect of ethics consultations on nonbeneficial life-sustaining treatments in the intensive care setting: a randomized controlled trial. 7AMA 2003;290:1166-72.

28. Lautrette A, Darmon M, Megarbane B, et al. A communication strategy and brochure for relatives of patients dying in the ICU. $N$ Engl 7 Med 2007;356: 469-78.

29. Ouimet S, Kavanagh BP, Gottfried SB, et al. Incidence, risk factors and consequences of ICU delirium. Intensive Care Med 2007;33:66-73.

30. Abelson J, Li K, Wilson G, et al. Supporting quality public and patient engagement in health system organizations: development and usability testing of the Public and Patient Engagement Evaluation Tool. Health Expect 2016;19: 817-27.

Affiliations: Departments of Critical Care Medicine (Krewulak, Sept, Stelfox, Fiest), Community Health Sciences (Stelfox, Ismail, Fiest) and Psychiatry (Ismail), O’Brien Institute for Public Health (Stelfox, Ismail, Fiest) and Hotchkiss Brain Institute (Ismail), Cumming School of Medicine, University of Calgary; Critical Care Strategic Clinical Network (Krewulak, Sept, Stelfox, Fiest), Alberta Health Services, Calgary, Alta.; Tennessee Valley Veterans Affairs Geriatric Research Education Clinical Center (Ely) and Critical Illness, Brain Dysfunction, and Survivorship Center (Ely), Vanderbilt University Medical Center, Nashville, Tenn.; Department of Education, Development and Research (Davidson), University of California, San Diego Health, San Diego, Calif.

Contributors: Bonnie Sept, Karla Krewulak, Kirsten Fiest and Henry Stelfox contributed substantially to the study conception and design, facilitated data acquisition and interpreted the data. Henry Stelfox, E.W. Ely, Judy Davidson and Zahinoor Ismail provided expert consultation. Karla Krewulak drafted the manuscript. All of the authors critically revised the manuscript for important intellectual content, approved the version to be published and agreed to be accountable for all aspects of the work.

Funding: This study was supported by grants to Kirsten Fiest from the Canadian Institutes of Health Research, the Calgary Centre for Clinical Research and the Critical Care Strategic Clinical Network.

Acknowledgements: The authors thank Doreen Rockliff, one of the patient partners, for her help in reviewing study materials and Krista Spence for her help with creating and piloting the delirium reference standard form. The authors also thank Israt Yasmeen, Danielle Demiantschuk and Brianna Rosgen for their help in enrolling participants.

Supplemental information: For reviewer comments and the original submission of this manuscript, please see www.cmajopen.ca/content/7/2/ E294/suppl/DC1. 